\title{
Automatic Control and Alert Message System for Intra Venous Fluid Level
}

\author{
T.Thangam, T. Murugajothi, R.Rajakumari, T.Suganya Thevi, M.Thiruveni
}

\begin{abstract}
The objective of this paper is to find a solution for the problem arising in IV drip bottles. We detected glucose levels in glucose trip bottles, which are used manually in hospitals. At the point when the glucose container is half vacant, an admonition message is given to send to the attendants working there. Three significant modules are utilized for this reason. Level sensor that can identify fluid levels in IV trickle bottles and is utilized as an info module used to send information to the controller. The PIC (Programmable Interface Controller) board is utilized as a controller module that procedures the information got from the sensor. The MGM module is utilized as a yield module dependent on the control given by the controller. It sends an alarm message to medical attendants' telephone numbers.The another one is, automatically the liquid flow in the tube will be stopped by using the solenoid valve, only when the liquid level in the drip bottles is about to minimum extend.
\end{abstract}

Keywords - Programmable Interface Controller, Arduino AT mega board, IV drip bottles;

\section{INTRODUCTION}

Hospitals utilize intravenous implantation treatment to treat patients. This mixture treatment utilizes a liquid or an answer that is regulated legitimately into the patient's vein. This imbuement treatment gives sustenance to a patient who doesn't be able to take nourishment from his mouth. It is likewise used to reestablish their lost liquid. A few patients who require imbuement treatment are those experiencing careful activity and those in an out cold condition, to give some examples. A portion of the advantages of the above drug are:

1. Delivers nutritional supplements and parental

2. Maintains and Restores the fluid and electrolyte balance .

Now and again, intravenous imbuement is given persistently, contingent upon the patient's condition and physician's instructions. This consistent mixture is called discontinuous imbuement or piggy implantation,it will be applied if a patient is getting medicine constantly. Intravenous implantation is a therapeutic term that portrays the way particular kinds of medications or different substances are appropriated in the body. Undeclared intravenous treatment may offer ascent to liquid over-burden or pneumonic blockage, which can slaughter a patient. The intravenous implantation method requires continuous

Revised Manuscript Received on October 15, 2019.

T.Thangam, Department of Electronics and Communication, PSNACET, Dindigul, Tamilnadu, India

T. Murugajothi, Department of Electronics and Communication, PSNACET, Dindigul, Tamilnadu, India(E-mail jothyece@ gmail.com)

R.Rajakumari, Department of Electronics and Communication, PSNACET, Dindigul, Tamilnadu, India

T.Suganya Thevi, Department of Electronics and Communication, PSNACET, Dindigul, Tamilnadu, India

M.Thiruveni, Department of Electronics and Communication, PSNACET, Dindigul, Tamilnadu, India observing dependent on the patient's condition and drug necessities. Be that as it may, in the foundation of the Philippine Hospital, a few doctors are as yet utilizing the manual procedure of checking and controlling the intravenous mixture system.

Following such a procedure, the restorative expert will expend a ton of time to consistently screen and control the degree of liquid in the IV pack. This manual procedure will interrupt the medical caretaker's undertaking and requires more faculty to cover these errands.

The point of the examination is to structure a control framework for intravenous piggy imbuement that will consistently screen and message the present circumstance to ward medical attendants/specialists. Explicit goals are:

1. To plan a control framework that can quantify and control the imbuement rate utilizing remote innovation,

2. To build up an Android application that can show the imbuement rate in drops every moment of the patient

3. Approves the information and tests the precision of the framework.

\section{STUDY OF EXISTING TECHNIQUES}

The device based on microcontroller that will control and monitor the flow of intravenous fluid (blood or glucose) injected in the body. Also, it will perform real-time monitoring of vital health parameters like heart beat rate, pulse rate and blood pressure and provide communication. Patient tracking structures[3] is the time period for all the numerous gadgets that are used to supervise sufferers. One classification of such gadgets is gadgets that pointers if the influenced individual gets directly into a basic state. In our proposed technique centers around to screen start alarm to specialists roughly the sufferers at certain purposes of glucose venture infusions. In our proposed gadget it will carried out four primary responsibilities, the progression of glucose will halted the buoy degree to be overseen, while the fluid degree underneath the verge cost will upward caution and cozy to comparing individual through remote, at that point the any infusions be applied methods it will be naturally infused relies upon the time based completely. Every one of the activities are controlled through microcontrollers. Furthermore, sensors are utilized to degree the cost of fluid, and diverse component basically based engine.

One technique contains IR sensor, RF transmitter, Receiver, signal, and so on. Fundamentally, IR transmitter sends an IR beam which is gotten by the Infra Red collector 
and deliberate yield is regarding voltage. At first, IR sensors (IR Transmitter - IR Receiver) are set at the base on either side of the saline container. The IR transmitter ceaselessly transmits the beams through the saline fluid and the IR beneficiary begins to get it and the comparing yield voltage is estimated as $4.5 \mathrm{~V}$. At the point when the drug fluid in the dribbles jug goes past the region secured by the IR sensor, the IR beneficiary gets a greater number of beams than in the past condition whose yield voltage is estimated as $5 \mathrm{~V}$. Another framework takes out the consistent manual checking of the degree of fluid in a jug is by utilizing the heap cell. The primary suggestion is given when $50 \mathrm{ml}$ of fluid is left so the medical clinic staff gets sufficient opportunity to arrive at the room and supplant the container. The subsequent insinuation is sent as a consider ready utilizing a GSM modem to show the earnest need to supplant the jug. Patient following structures [5] is the timespan for all the various contraptions that are utilized to administer sufferers. One class of such gadgets is gadgets that pointers if the influenced individual gets directly into a basic state. In our proposed technique centers around to screen start alarm to specialists roughly the sufferers at certain purposes of glucose venture infusions. In our proposed gadget it will carried out four principle responsibilities, the progression of glucose will halted the buoy degree to be overseen, while the fluid degree underneath the edge cost will upward caution and cozy to comparing individual through remote, at that point the any infusions be applied methods it will be consequently infused relies upon the time based completely. Every one of the activities are controlled through microcontrollers. Also, sensors are utilized to degree the cost of fluid, and diverse component fundamentally based engine.

\section{PROPOSED TECHNIQUES AND ITS DESCRIPTION}

According to our investigation, we found a strategy to use voice alert but this technique only works when the patient concerned will be in a cognitive state. However, it is consistently inconceivable. Due to this voice, close to patients also get upset. Therefore we search for an alternative technique to maintain a strategic distance from this issue. We found a strategy which looked at the degree of glucose in glucose trickle bottles used in medical clinics. At this point when the glucose container is being halved, an alarm message is sent to the medical attendants. At this point when the glucose levels in the glucose excursion bottles used in the clinic are running out, an alarm message is sent to the medical carers working there. Burden sensors can measure weight are used tool for information modules used to send information to the microcontroller. The super board which is of Arduino ATmodel is used as a module for controller that processes the information obtained from the sensor. The Global System for Mobile Communication module is used as a yield module that relies on the direction given by the Microcontroller, the medical attendant.

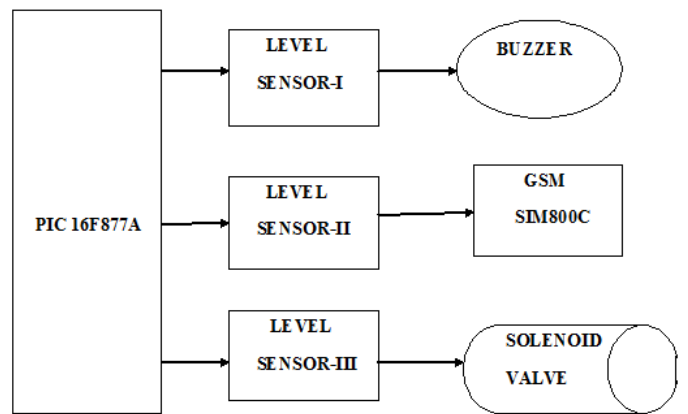

Fig.1.General Block diagram

The sensors used include two types of yield, for example, rearranging yield and non-converting yield. The load cell of S-type is hung in the holder of glucose and the container in the S-type load cell is hung on the opposite end. In this manner the heaviness of the estimated container is transformed to the appropriate voltage and henceforth the yield as of the heap cell as the voltage and the voltage altitude is raised to a specific altitude and heap cell nonconverted yield is given as follows. Contribution to one of the Pulse width Modulated sticks in the ATMMA. The yield of the PWM pin will vary from 0-255. We apply stack cell expansion from $0-750 \mathrm{~g}$. The most extreme estimate of the PWM Pins Esteem would be 255 when the heap cell can gauge its greatest range. This method is called scaling.

A GSM based programmed cautioning and showing gadget is proposed where a weight sensor is utilized as a level sensor .It depends on the key that the IR sensor yield voltage level changes when intravenous liquid level is beneath sure limit. A comparator is utilized to constantly come close the message yield with predefined edge. When the handset yield is negative then the Arduino controller recognizes the liquid level is excessively low and it cautions the eyewitness through the mounted LCD show and the cell phone at the control room demonstrates the room number of the patient for snappy recuperation.

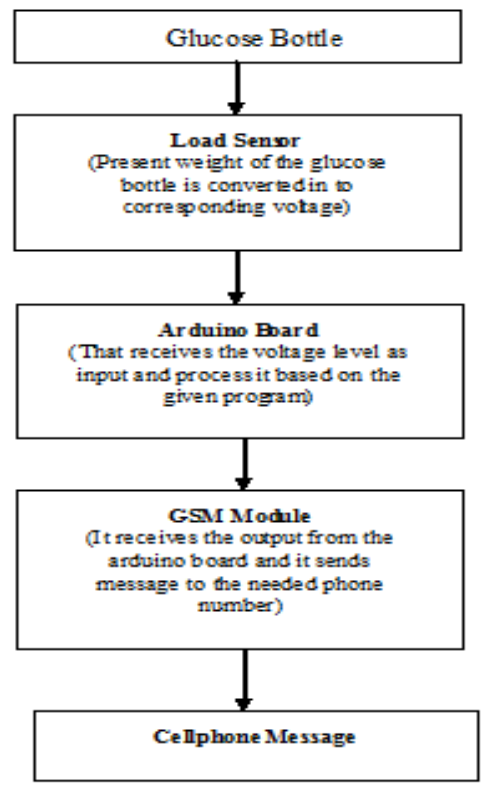

Fig.2.Flowchart shows functional description 
The largest weight of glucose bottle for our application is 500 which is equivalent to 170 in PWM yield. Once the jug is completed along these lines the PWM stick yield will attain 170 when the module of GSM will transmits the message as "Container is full". If there is a constraint in estimating the glucose level, the level of the container is continuously estimated. The GSM module will send the message "Check mechanical assembly". At the time when the glucose level falls on a large part of the PWM stick yields 85 , the GSM module will send the message because "jaggery is halved". In addition, when PWM production is $17 \%$, the glucose level falls to 17 , becoming a distress warning and an alarm message is sent as "distress, container reduced by $10 \% "$. Power supply for Global System for Mobile Communication modules. Characteristics of the glucose replacement scheme are shown in Table 1.

\begin{tabular}{|c|c|c|c|}
\hline S.No & Weight & $\begin{array}{c}\text { PWM } \\
\text { Values }\end{array}$ & Message \\
\hline 1 & $500 \mathrm{~g}$ & 170 & The bottle is full \\
\hline 2 & $250 \mathrm{~g}$ & 85 & $\begin{array}{c}\text { The bottle is } \\
\text { reduced to } 50 \%\end{array}$ \\
\hline 3 & $50 \mathrm{~g}$ & 17 & $\begin{array}{c}\text { Emergency, the bottle } \\
\text { is reduced to } 10 \%\end{array}$ \\
\hline
\end{tabular}

Table.1. Characteristics of glucose level

\section{COMPONENTS USED}

\section{(1) MICRO CONTROLLER PIC16F877A:}

The PIC16F group of gadgets is CMOS (Complementary Metal Oxide Semiconductor). CMOS innovation offers various points of interest over different advancements. For instance, CMOS circuits devour next to no power, work over a serious wide voltage run and are very sympathetic of awful design and electrical commotion. The name PIC at first alluded to "Fringe Interface Controller".

\section{(2) BUZZER:}

The buzzer is a sound device, which can be electromechanical ,mechanical or piezoelectric. Initial device dependent on structure indistinguishable from the electric ringer with no use of metal gong. Substantially, a hand off may be accomplished with an intrusion with its active current, causing the contact to swell. Often these units were protected to a divider to be used as a Audio board. The "buzzer" from the scratching clamor is the electromechanical buzzer.

\section{(3) SOLENOID VALVE}

A solenoid valve which is of electromagnet is the operated valve.It is constrained by an current pass along a solenoid: in the example of a 3-port valve, outpotting is exchanged between two outlet ports. The museum solenoid valve can be set simultaneously but Complicated.

Solenoid valves in liquids are the control components used every now and then. Liquids are to stop, release, part, circulate, or mixture liquids. They are used in many new application areas. Solenoids provide quick exchange, highly reliable, long life span, great moderate uniformity of materials which is used, low power consumption and little planning.

\section{(4) LEVEL SENSOR}

Level sensors found the fluid degree and various liquids as well as liquefied solids, including powders and granular materials that provides an upper free surface. Level estimates may either be in vain or may be Esteem. Constant level sensors inside a predefined extension decide the measurement level and fixed measurement of a material in a specific location, Whereas the point-level sensors will display whether the material is above or below the Threshold point. For the most part the final detection levels which are extremely high or low.

\section{RESULTS AND DISCUSSION}

Each part that we are to be applied for use in the glucose level pointer is purchased and tried independently to find out if every module is functioning correctly. The circuit which is used as a load sensor that provides a shifted yield that ranges from $0-10 \mathrm{mV}$. Currently detecting module is finished. At that point the ARDUINO board is first tried to establish some data sources and programming for sticks and some other native applications. The yield from the heap sensor at that point is given as a contribution from the ARDUINO board and the programming part designed in such a way that was embodied in the board at that point so that as soon as we are informed, the corresponding required yield stick is some exceptional result. And subsequently the board of ARDUINO is tried independently along the correct program. GSM modules are tried independently at that point. For the first few other direct applications, it is inspected and 3-pins accessible in the given module and joined to the yield from the ARDUINO board. For the program which is provided and possible steps it effectively transmit the messages to the telephone number provided in the given program and the module is additionally tried. Accordingly the whole module set is tried and yield has been achieved.

\section{V.CONCLUSION}

In our undertaking we have built up a keen IV dribble unit which works at a low power. It has better productivity contrasted with the current framework and the proposed strategy will decrease the minor mishaps in medical clinics (for example reverse of blood, liquid maintenance, blood misfortune). In our task we sets three sorts of cautions such, as ringer sound, ready messages utilizing GSM, solenoid valve for naturally shut the fluid stream when the glucose jug is going to be purged. When this glucose level marker appears the individuals dealing with the patients and their concern care taker no need to stress over the time that will consume for the glucose container to discharged. At the point when Glucose container level comes underneath $30 \%$,Since our message notice framework send the alarm messages to medical caretakers, they can go to the cases at the perfect duration and supplant the bottle of glucose before that gets totally exhausted. Therefore it helps medical caretakers and patients in the emergency clinic and make 
them not to stress over anything and do their work with no pressure. Therefore our undertaking ideally a wellbeing amicable and observing gear, can be utilized in the emergency clinic to improve quiet wellbeing.

\section{REFERENCES}

1 Pradeep kumar k,"Autonomous intravenous infusion system \& health monitoring "published in asar international conference,may-2014.

2 Paul Bustamante," A new wireless sensor for intravenous dripping detection"Publised in International Journal on Advances in Networks and Services (IJANS), vol.3, 2010.

3 Ram kumar.S,"Remote monitoring the glucose bottle level in hospitals" Published in International Conference on Emerging Trends in Application of Computing (ICETAC), 2017.

4 C.S.Sundar Ganesh, "automatic indication system of glucose level in glucose trip bottle "Published in International Journal Of Multidisciplinary Research and Modern Education(IJMRME) vol.3,2017.

5 Arjun udayan,"A survey on automatic flow control in drip "Published in International Journal OF Advanced Research in Electrical, Electronics and Instrumentation Engineering (IJAREEIE), vol.5, March 2016.

6 Bailey Flynn, Matthew Matthew Nojoomi, Michael Pan, Kamal Shah, Erica Skerrett, "IV DRIP: Intravenous Dehydration Relief in Pediatrics", instituteof global health technology.

7 K.J. K.J. Ayala, "The 8051 Microcontroller “, 3rd Edition, Microcontrollers Pros Corporation, 2004. 5. simon monk, "programming arduino".

8 www.circuitstoday.com 